\title{
Evolving therapies in the treatment of hepatocellular carcinoma
}

\author{
Hans Christian Spangenberg \\ Robert Thimme \\ Hubert E Blum \\ Department of Medicine II, University \\ of Freiburg, D-79106 Freiburg, \\ Germany
}

\begin{abstract}
Hepatocellular carcinoma (HCC) is one of the most common malignant tumors worldwide. The major etiologies and risk factors for HCC development are well defined and some of the steps involved in hepatocarcinogenesis have been elucidated in recent years. Therapeutic options that can be applied in curative or palliative intention are available and are dependent on the HCC stage. The therapeutic options fall into five main categories: (1) surgical interventions, including tumor resection and liver transplantation, (2) percutaneous interventions, including ethanol injection and radiofrequency thermal ablation, (3) transarterial interventions, including embolization and chemoembolization, (4) radiation therapy, and (5) drugs as well as gene and immune therapies. Until recently, no therapy existed for patients with advanced HCC. In 2007 a multikinase inhibitor (sorafenib) showed for the first time a significant increase in overall survival in patients with advanced HCC. Furthermore, several other agents that target different factors of hepatocarcinogenesis (eg, epidermal growth factor, insulin-like growth factors, hepatocyte growth factor, vascular endothelial growth factor, fibroblast growth factor, platelet-derived growth factor, and the transforming growth factors- $\alpha$ and $-\beta$ ), have emerged and been tested in clinical trials. This review gives an overview of the current therapeutic strategies and their clinical impact.
\end{abstract}

Keywords: hepatocellular carcinoma, targeted therapy, sorafenib, antiangiogenesis

\section{Introduction}

Hepatocellular carcinoma (HCC) is one of the most common malignant tumors worldwide (Okuda 2000; Bruix et al 2001, 2004; Befeler and Di Bisceglie 2002; Bruix and Llovett 2002; Llovet et al 2003, 2004; El-Serag 2004; Fattovich et al 2004; Kiyosawa et al 2004; Serman and Klein 2004) and has been reviewed recently (Sherman and Klein 2004; Bruix and Sherman 2005; Marrero 2006; El-Serag and Rudolph 2007; Kulik 2007). The incidence ranges from $<10$ cases per 100,000 population and year in North America and Western Europe to 50-150 cases per 100,000 population and year in parts of Africa and Asia where HCC is responsible for a large proportion of cancer deaths. However, a rise in the incidence of and mortality from HCC, most likely reflecting the high prevalence of hepatitis $\mathrm{C}$ virus (HCV) infection, has also been observed in most industrialized countries (Defuffic et al 1998; El-Serag and Mason 1999; El-Serag 2004; Capocaccia et al 2007; Taylor-Robinson et al 1997).

\section{HCC etiologies and risk factors}

The major etiologies of HCC are well defined (Table 1) and include, apart from the well known factors, overweight in men (Calle et al 2003), non-alcoholic fatty liver disease (NAFLD), and diabetes mellitus (El-Serag 2004). The HCC risk in patients with liver cirrhosis depends on the activity, duration, and the etiology of the underlying liver disease (Figure 1). Clinical and biological variables, eg, age, anti-HCV positivity, partial prothrombin time, and platelet count, enable further 
Table I Major hepatocellular carcinoma etiologies

Chronic hepatitis B, C, and D
Toxins (eg, alcohol, tobacco, aflatoxins)
Hereditary metabolic liver diseases (eg, hereditary hemochromatosis,
$\alpha$-I-antitrypsin deficiency)
Autoimmune hepatitis
States of insulin resistance
Overweight in males
Diabetes mellitus
Non-alcoholic steatohepatitis (NASH) or non-alcoholic fatty liver
disease (NAFLD)

indentification of a subset of patients with the highest risk of HCC development (Thomas et al 2007). While patients with hepatitis e antigen ( $\mathrm{HBeAg}$ ) positive chronic hepatitis $\mathrm{B}$ virus (HBV) have a higher risk of HCC development (Yang et al 2002) and of early recurrence and poor survival after curative resection (Sun et al 1997) than anti-HBe positive patients, a recent study in Taiwanese patients showed that the HBV DNA concentration in serum (viral load) correlates with the HCC risk, independent of the $\mathrm{HBeAg} /$ anti-HBe status and the aspartate aminotransferase or alanine aminotransferase level (Chen et al 2006). Also, occult (anti-HBc positive only) and past HBV infection (anti-HBc and anti-HBs positive) carry higher HCC risk than healthy individuals (Yano et al 2002; Pollicino et al 2004). For HCV infected patients, recently identified viral risk factors for HCC development are genotype $1 \mathrm{~b}$ (Bruno et al 2007) and amino acid substitutions in the HCV core region (Akuta et al 2007).

Importantly, coexistence of etiologies, eg, HBV and $\mathrm{HCV}$ infection, $\mathrm{HBV}$ infection, and aflatoxin $\mathrm{B} 1$ exposure

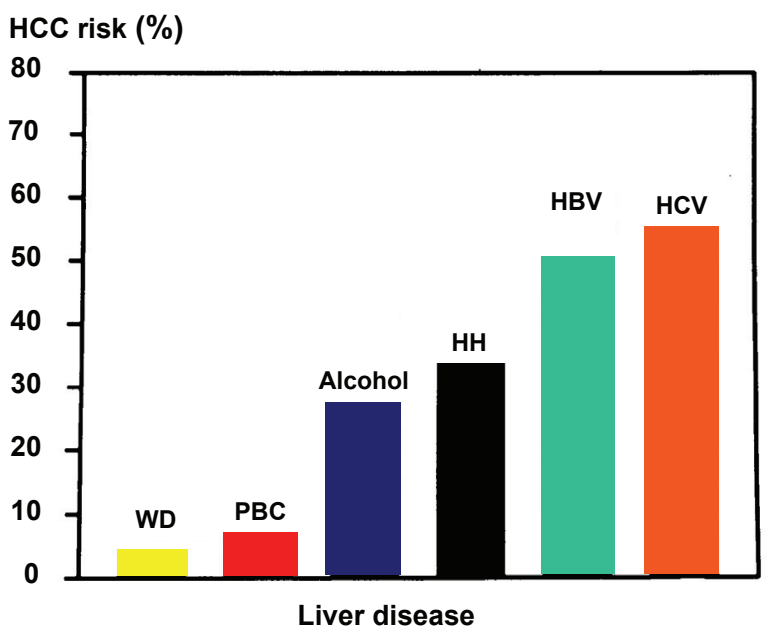

Figure I Hepatocellular carcinoma risk in liver cirrhosis from different etiologies. Abbreviations:WD,Wilson's disease; $\mathrm{PBC}$, primary biliary cirrhosis, $\mathrm{HH}$, hereditary hemochromatosis; $\mathrm{HBV}$, hepatitis $\mathrm{B}$ virus infection; $\mathrm{HCV}$, hepatitis $\mathrm{C}$ virus infection.
(Yu and Yan 2004; Ming et al 2002), HBV or HCV infection and alcohol use or diabetes mellitus (Hassan et al 2002), HCV infection and liver steatosis (Ohata et al 2004), environmental factors, eg, alcohol (Morgan et al 2004; Yu and Yan 2004; Marrero et al 2005b), as well as diabetes mellitus, obesity, and tobacco use (Calle et al 2003; Caldwell et al 2004; El-Serag et al 2004; Marrero et al 2005b), has to be considered and further increases the relative risk of $\mathrm{HCC}$ development. Interestingly, coffee consumption appears to reduce the HCC risk (Bravi et al 2007). HCC is generally more frequent in males than in females. Whether this is related to the protective effect of estrogens, as recently demonstrated in an animal model of chemical hepatocarcinogenesis (Naugler et al 2007), remains to be determined.

\section{HCC pathogenesis}

Some of the steps involved in the molecular pathogenesis of HCC have been elucidated in recent years (El-Serag and Rudolph 2007). As for most types of cancer, hepatocarcinogenesis is a multistep process involving different genetic alterations that ultimately lead to malignant transformation of the hepatocyte. While significant progress has been made in recognizing the sequence of events involved in other forms of cancer, most notably in colorectal cancer and certain hematopoietic malignancies, the molecular contribution of the different factors and their interaction in hepatocarcinogenesis are still poorly understood. HCC is phenotypically (morphology, microscopy) and genetically very heterogenous, possibly in part due to the heterogeneity of etiologic factors implicated in HCC development, the complex functions of the liver cell, and the advanced stage at which HCC usually are becoming clinically symptomatic and diagnosed. Malignant transformation of hepatocytes may occur regardless of the etiologic agent through a pathway of increased liver cell turnover, induced by chronic liver injury and regeneration in a context of inflammation, immune response, and oxidative DNA damage. This may result in genetic alterations that cause activation of cellular oncogenes or inactivation of tumor suppressor genes (Dufour et al 2007), possibly in cooperation with genomic instability, including DNA mismatch repair defects and impaired chromosomal segregation, overexpression of growth and angiogenic factors, and telomerase activation (Ozturk 1999; Brechot 2004; Satayanarayma et al 2004; Suriawinata and Xu 2004; Yu and Yan 2004). Further, epigenetic modifications, eg, aberrant methylation, seem also to be involved in the molecular pathogenesis of human HCC (Calvisi et al 
2007). Chronic hepatitis B, C, and D, alcohol, metabolic liver diseases such as hemochromatosis and $\alpha$-1-antitrypsin deficiency, as well as NAFLD may act predominantly through this pathway of chronic liver injury, regeneration, and cirrhosis. Since the vast majority of HCC (70\%-90\%) develop in a cirrhotic liver, liver cirrhosis is a major clinical risk factor for HCC development. Most HCC occur after many years or decades of chronic hepatitis that provides the mitogenic and mutagenic environment that induces random genetic alterations which eventually result in the malignant transformation of hepatocytes and HCC development. While there is evidence that HBV and possibly also HCV may under certain circumstances play an additional direct role in the molecular hepatocarcinogenesis, aflatoxins have been shown to induce mutations of the p53 tumor suppressor gene, thus pointing to the contribution of an environmental factor to tumor development at the molecular level. Further, in a transgenic mouse model it has been shown that chronic immune-mediated liver cell injury without environmental or infectious agents is sufficient to cause HCC (Nakamoto et al 1998, 2002) and that inhibition of cytotoxic T lymphocyteinduced apoptosis and chronic inflammation by neutralization of the Fas ligand prevents HCC development in this model (Nakamoto et al 2002). In addition, also in a transgenic mouse model it has been demonstrated that NF-kappa B may be the link between inflammation and HCC development (Balkwill and Coussens 2004; Pikarsky et al 2004). Finally, individual polymorphisms of drug metabolizing enzymes, eg, various cytochrome $\mathrm{P} 450$ oxidases, $\mathrm{N}$-acetyltransferases, and glutathione-S-transferase, may contribute to the genetic susceptibility to HCC development (Chen and Chen 2002).

\section{HCC staging}

For HCC staging, seven systems have been proposed that address the extent and prognosis of the disease (Di Bisceglie 2004; Talwalker and Gores 2004): the Okuda staging system (Okuda et al 1985), the TNM classification and its modification by the Union International Contre Cancer (UICC) (Leung et al 2002), the Barcelona Clinic Liver Cancer (BCLC) classification (Llovet et al 1999), the Cancer of the Liver Italian Program (CLIP) score (Farinati et al 2000), the Japan Integrated Staging (JIS) score (Kudo et al 2004), the Groupe d'Etude de Traitement du Carcinoma Hepatocellulaire (GRETCH) score (Chevet et al 1999), and the Chinese University Prognostic Index (CUPI). The Okuda staging system is very effective for the identification of a subgroup of patients (Okuda III) with a very poor prognosis who should be treated with best supportive care (BSC) only. The BCLC classification appears especially useful for the selection of treatment options but has not been independently validated. The CLIP score was shown to be superior to the Okuda staging system but has not been systematically assessed in patients undergoing resection or liver transplantation. While a recent study indicates that the new prognostic JIS score is

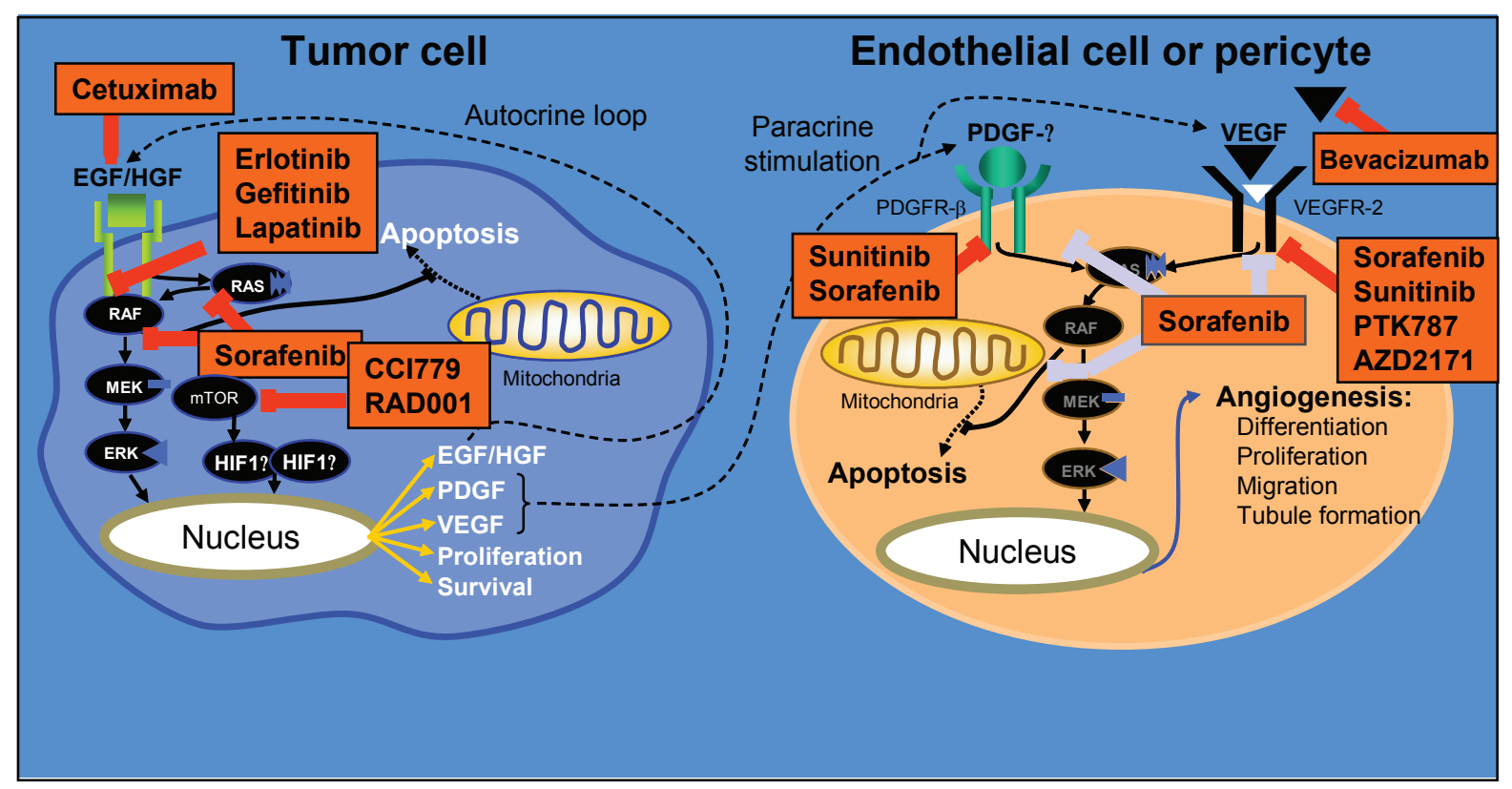

Figure 2 Key pathways in hepatocarcinogenesis and molecularly targeted agents currently under investigation in treatment of advanced HCC. Adapted with permission from Gollob JA, Wilhelm S, Carter C, et al. 2006. Role of Raf kinase in cancer: therapeutic potential of targeting the Raf/MEK/ERK signal transduction pathway. Semin Oncol, 33:392-406. Copyright (C) 2006 Elsevier. 
superior to the CLIP score (Kudo et al 2004), a comparison of the staging systems in an American cohort revealed that the BCLC had the best independent predictive power (Marrero et al 2005a).

The natural course of the disease and the median survival of patients with HCC depend on the stage of the disease at the time of diagnosis. In patients with CLIP score 0 or Okuda stage I the median survival is in the range of 23-69 months, while in patients with CLIP score $3-5$ or Okuda stage III median survival is only 1-14 months (Befeler and Di Bisceglie 2002). The staging system is clinically most important for the appropriate choice of the therapeutic strategy for individual patients. Cirrhotic patients developing a HCC during the last 5 years of surveillance survived longer than previously, due to improved management of the tumor and of the complications of cirrhosis (Sangiovanni et al 2004). Importantly, however, in a population-based study in the US underutilization of potentially curative therapies even among patients with favorable $\mathrm{HCC}$ features is a problem that needs to be addressed (El-Serag et al 2006).

\section{Current therapeutic strategies for HCC}

Current therapeutic strategies for HCC can be devided into established therapies such as surgical interventions (tumor resection and LTx), percutaneous interventions (ethanol injection, radiofrequency thermal ablation), transarterial interventions (embolization, chemoperfusion, or chemoembolization), and experimental strategies such as radiation therapy and drugs, including gene and immune therapy. Potentially curative therapies are tumor resection, LTx, and percutaneous interventions that can result in complete responses and improved survival in a high proportion of patients. In selected cases transarterial interventions result in palliation with, in some cases, good response rates and improved survival. Until recently, for patients with advanced HCCs no therapy was available that prolonged overall survival (OS), indicating the need for new targeted-therapies. In 2007 Sorafenib, a multikinase inhibitor, showed an increase of overall survival in patients with unresectable HCC. Besides this agent, various different molecules have been engineered that are currently tested in various tumors including HCC. These targeted therapies are reviewed below.

\section{Potential targets for HCC therapies}

Growth factors, their related receptors, and downstream signaling pathways play a major role in the development and maintenance of various cancers including $\mathrm{HCC}$ and are of significant interest for future therapeutic approaches. During fetal life, a large number of growth factors including the epidermal growth factor (EGF), insulin-like growth factors (IGFs), the hepatocyte growth factor (HGF), the vascular endothelial growth factor (VEGF), the fibroblast growth factor (FGF), the platelet-derived growth factor (PDGF), and the transforming growth factors- $\alpha$ and $-\beta$ (TGF- $\alpha$, TGF- $\beta$ ) are produced in the liver. Their secretion declines or shuts down in an adult liver; however, when liver regeneration is required after injury or damage (Diehl 2002; Duncan 2003), particularly growth factors such as EGF, TGF- $\alpha$, IGFs, and VEGF can be upregulated by normal hepatocytes. This normally transient upregulation is dysregulated in the chronic injured liver leading to sustained mitogenic/ oncogenic signaling, thereby playing an important role in hepatocarcinogenesis.

Furthermore, members of the FGF and PDGF families play important roles in promoting liver fibrosis and $\mathrm{HCC}$ growth (Ogasawara et al 1996; Campbell et al 2005). Like HGF, these growth factors are produced and released from non-hepatocyte sources such as activated hepatic stellate cells, myofibroblasts, endothelial cells, Kupffer cells, and bile duct epithelia and do also contribute to hepatocarcinogenesis.

Among the most critical cellular signaling pathways that support hepatocarcinogenesis are the receptor tyrosine kinase-activated pathways which include the rat sarcoma/rat sarcoma-activated factor (raf)/mitogen activated protein kinase/extracellular regulated kinase kinase/extracellular regulated kinase pathway (Ras/Raf/MEK/ERK), the Janus kinase/signal tranducers and activator of transcription pathway (JAK/STAT)s, and the phosphatidylinositide 3 kinase/protein kinase $\mathrm{B}$ (AKT)/mammilian target of rapamycin pathway (PI3K/AKT/mTOR) (Hopfner et al 2008; Zhu 2008).

These factors represent potential therapeutic targets in HCC treatment (Table 2). A selection of agents currently in the development and/or testing stages for the clinical application in targeted HCC treatment is summarized in the following section.

\section{Anti-EGFR therapies}

The expression of several EGF family members, specifically EGF, TGF- $\alpha$, and heparin binding-EGF, as well as the EGFR, has been described in several HCC cell lines and in tissue. Multiple strategies to target EGFR signaling pathways have been developed, and two classes of anti-EGFR agents display antitumor activity in cancer: monoclonal 
Table 2 Selection of agents for targeted therapy in hepatocellular carcinoma

\begin{tabular}{|c|c|c|}
\hline Name & Target & Current status \\
\hline Gefitinib & EGFR & $\begin{array}{l}\text { phase } 2 \text { study (O'Dwyer et al } \\
\text { 2006) }\end{array}$ \\
\hline Erlotinib & EGFR & $\begin{array}{l}\text { phase } 2 \text { study (Philip et al } \\
\text { 2005; Thomas et al 2007) }\end{array}$ \\
\hline Lapatanib & EGFR & $\begin{array}{l}\text { phase } 2 \text { study (Ramanathan } \\
\text { et al 2006) }\end{array}$ \\
\hline Cetuximab & EGFR & $\begin{array}{l}\text { phase } 2 \text { study (Gruenwald et al } \\
2007 \text { Louafi et al 2007; Zhu } \\
\text { et al 2007) }\end{array}$ \\
\hline Bevacizumab & VEGF & $\begin{array}{l}\text { phase } 1 / 2 \text { study (Schartz et al } \\
2006 \text { Thomas et al 2007) }\end{array}$ \\
\hline Sorafenib & $\begin{array}{l}\text { c-Rafl, B-Raf, } \\
\text { VEGFR PDGFR }\end{array}$ & $\begin{array}{l}\text { phase } 3 \text { study (Llovet et al } \\
\text { 2007) }\end{array}$ \\
\hline Sunitinib & $\begin{array}{l}\text { PDGFR,VEGFR, } \\
\text { c-KIT, FLT-3 }\end{array}$ & $\begin{array}{l}\text { phase } 2 \text { study (Faivre et al } \\
\text { 2007; Zhu et al 2007) }\end{array}$ \\
\hline Vatalanib & $\begin{array}{l}\text { VEGFR, PDGFR, } \\
\text { c-KIT }\end{array}$ & $\begin{array}{l}\text { phase I study (Koch et al } \\
\text { 2005) }\end{array}$ \\
\hline Cediranib & VEGFR & $\begin{array}{l}\text { phase } 2 \text { study (Alberts et al } \\
\text { 2007) }\end{array}$ \\
\hline Rapamycin & mTOR & $\begin{array}{l}\text { phase I/2 study (Sahin et al } \\
\text { 2004; Rizell et al 2005) }\end{array}$ \\
\hline Everolimus & $\mathrm{mTOR}$ & $\begin{array}{l}\text { phase I/2 study (Sahin et al } \\
\text { 2004; Rizell et al 2005) }\end{array}$ \\
\hline Bortezomib & proteasome & $\begin{array}{l}\text { Phase I/2 study (Hegewisch- } \\
\text { Becker et al 2004) }\end{array}$ \\
\hline
\end{tabular}

antibodies (eg, cetuximab), which competitively inhibit extracellular endogenous ligand binding, and small molecules, which inhibit the intracellular TK domain (eg, gefitinib, erlotinib).

While in vitro and in vivo studies with these agents have been shown to inhibit proliferation of HCC and metastasis formation, only a few clinical studies have been conducted so far. Two phase 2 clinical studies have evaluated the safety and efficacy of erlotinib given at a dose of $150 \mathrm{mg}$ daily in patients with advanced HCC. In the study by Philip et al (2005), 39\% of patients had a partial response, and 32 had stable disease at 6 months. Median OS was 13 months. In a study by Thomas et al (2007), 43\% of patients achieved progression-free survival (PFS) at 16 weeks. Median OS was 25.0 weeks. Another compound, gefitinib, given at $250 \mathrm{mg}$ daily was examined in a single-arm phase 2 study. Thirtyone patients were recruited for the study (O'Dwyer et al 2006). One patient had a partial response, and 7 patients had stable disease. Median PFS was 2.8 months, and the median OS was 6.5 months. Lapatinib, a selective dual inhibitor of both EGFR and HER-2/NEU TKs, also demonstrated modest activity in $\mathrm{HCC}$ in a preliminary report (Ramanathan et al 2006).

Cetuximab was tested in two studies in patients with advanced HCC. Zhu et al (2007) enrolled 30 patients with advanced HCC. Five patients had stable disease, median OS was 9.6 months, and the median PFS was 1.4 months. Gruenwald et al (2007) reported their experience of cetuximab in a similarly designed study and observed no responses in 27 patients; the median time to progression for all patients was 8 weeks.

The combination of cetuximab with GEMOX was evaluated in a phase 2 study (Louafi et al 2007). Forty-three patients were enrolled and 35 patients were available for efficacy analysis with a response rate of $23 \%$.

Currently, further anti-EGFR-based approaches are being tested in clinical trials, especially in combination with either conventional cytostatics or with other targeted agents (Heuther et al 2005a, b; Moore et al 2005; Bourhis et al 2006) in order to block several carcinogenic pathways.

\section{Antivascular endothelial growth factor/vascular endothelial growth factor receptor agents}

HCCs are tumors with high vascular architecture. VEGF is a key factor in tumor angiogenesis and high levels of VEGF have been associated with the HCC grade and clinically with inferior OS (Chao et al 2003; Jeng et al 2004; Poon et al 2004). Therefore, the inhibition of angiogenesis represents a potential and promising therapeutic target in HCC. Bevacizumab, a humanized murine monoclonal anti-VEGF antibody, alone or in combination with standard chemotherapy has become standard treatment in several human cancers (eg, colon cancer). Studies with bevacizumab monotherapy and in combination with other agents have been performed in patients with advanced HCC. Most of the trials were phase 2 studies with encouraging results for PFS and OS. Of interest is the study by Thomas et al (2007). In this phase 2 study, bevacizumab at a dose of $10 \mathrm{mg} / \mathrm{kg}$ intravenously once every 14 days and erlotinib at a dose of $150 \mathrm{mg}$ orally daily was given to patients with advanced HCC (Thomas et al 2007). Thirty-four patients were eligible for the efficancy analysis: 1 patient had a confirmed complete response (CR), and 6 patients had a partial response (PR), for a 21\% response rate. Median PFS was 9 months, and median OS 19 months. However, these promising results have to be confirmed in a randomized controlled study.

In terms of safety and tolerability, despite the initial safety concerns for bevacizumab use in patients with liver 
cirrhosis and portal hypertension, in particular the risks for gastrointestinal bleeding and thrombosis, the most common bevacizumab-related side effects were hypertension, bleeding, and proteinuria, that were generally well managed (Schwartz et al 2006).

In addition, several agents that inhibit the tyrosine kinase activity of VEGFR have been synthesized. PTK787/ ZK222584 (vatalanib) is an oral angiogenesis inhibitor that targets all known VEGFR TKs. In an open-labeled, multicenter, phase 1 study the safety, tolerability, and pharmacokinetic profile of PTK787 with a daily dose between $750 \mathrm{mg}$ to $1250 \mathrm{mg}$ was analyzed in patients with unresectable HCC (Koch et al 2005). $750 \mathrm{mg}$ daily was defined as maximal tolerated dose; however, in terms of efficacy no complete response or partial response was observed. Fifty percent of the analyzed patients had stable disease and 50\% had progressive disease.

Another potent, oral, pan-VEGFR TK inhibitor with activity against PDGFRs and c-Kit is AZD2171 (cediranib). So far, one phase 2 study by Alberts et al (2007) has studied the administration of AZD2171 (45 mg daily for a 28-day treatment cycle) in patients with advanced HCC. In terms of toxicity, $84 \%$ developed grade 3 toxicity mainly fatigue, hypertension, and anorexia.

A further encouraging approach is the use of dualtargeting tyrosine kinase inhibitors, such as NVP-AEE788 or zactima (ZD6474). These molecules target both the VEGFRs and the EGFR. Recently, in in vivo studies of non-HCC tumor models (eg, colon, cholangiocarcinoma, NSCLC), NVPAEE788 showed significant antitumor efficacy based on the inhibition of tumor cell proliferation by blocking hepatoma EGFR and angiogenesis by blocking endothelial VEGFR. Nevertheless, an evaluation of this interesting observation in clinical trials is warranted (Heymach 2005; Busby et al 2006; Wiedmann et al 2006; Younes et al 2006.

\section{Multi-kinase inhibition}

Hepatocarcinogenesis is a complex process involving various, diverse signaling pathways. Therefore, molecularly targeted agents that interact with multiple signaling pathways/effectors appear to be very attractive in the treatment of patients with HCC. The novel bi-aryl urea sorafenib $\left(\right.$ Nexavar $\left.^{\circledR}\right)$, an orally available multi-kinase inhibitor, targets kinases of wild-type B-Raf, mutant V559EB-Raf and CRaf, thereby blocking tumor growth. In addition, sorafenib inhibits receptor tyrosine kinases involved in angiogenesis, including human VEGF receptors-2 and -3 (VEGFR-2/-3) and PDGF- $\beta$ R.
Sorafenib has been tested for the treatment of advanced HCC. In a phase 2 trial 137 patients with advanced, inoperable HCC, sorafenib (400 mg bid) induced a partial response in $2.2 \%$, a minor response in $5.8 \%$ and a stable disease (SD) in $34 \%$ of patients (Abou-Alfa et al 2006). The median time to progression (TTP) was 4.2 months, and the median OS was 9.2 months. Sorafenib was fairly well tolerated. Grade 3 and 4 drug-related toxicities were fatigue (9.5\%), diarrhea (8\%), and hand-foot skin reaction (5.1\%). Interestingly, in an analysis of a subgroup of treated patients $(n=33)$, pretreatment tumor phosphorylated ERK (pERK) levels were correlated with the TTP. Patients with an intense pERK staining in their tumors survived longer, suggesting that inhibition of the Raf/MEK/ERK pathway is central to sorafenib's mode of anti-tumor action in HCC (Fattovich et al 2004). Whether this generally holds true for HCC remains to be determined. In other tumor entities the antineoplastic potency of sorafenib appears to be mainly due to its antiangiogenic activity (Wilhelm et al 2004; Liu et al 2006).

Based on the encouraging, positive results of this phase 2 trial, a randomized, double-blinded, phase 3 trial with 602 patients with advanced HCC was initiated. An evaluation of this international study (Sorafenib HCC Assessment Randomized Protocol [SHARP]) led to discontinuation, as the HCC patients treated with sorafenib achieved a significant survival benefit over the placebo-treated controls. The data of the analysis were presented at the ASCO meeting in 2007 and showed that median OS in the sorafenib-treated arm was 10.7 months vs 7.9 months in the control arm (Llovet et al 2007). Of note, the median TTP was 5.5 months in the sorafenib arm vs 2.8 months in the control arm. The toxicity profile of this trial was comparable with that of the phase 2 trial. Based on these findings, sorafenib represents the first agent that has shown improved OS benefits in patients with advanced HCC and has recently gained accelerated approval by the FDA for the treatment of advanced unresectable HCC.

Another interesting multikinase inhibitor is sunitinib $\left(\right.$ Sutent $\left.^{\circledR}\right)$. It is a small molecule that inhibits members of the split-kinase domain family of receptor TKs (RTKs) including VEGFR types 1 and 2 (fms-related TK 1 [FLT1] and the FLK1 kinase insert domain receptor [FLK1/KDR]); PDGFR- $\alpha$ and PDGFR- $\beta$, the stem cell factor receptor c-KIT, and the FLT3 and RET kinases (Pawson 2002; Mendel et al 2003; Arora et al 2005). In vitro studies with various tumor cell lines have shown that the antiangiogenic effects of sunitinib are mediated through VEGFR and PDGFR. Clinically, sunitinib has good oral bioavailability and is approved for 
the treatment of renal cell carcinoma and gastrointestinal stromal tumor.

Two independent phase 2 studies have examined the tolerability and efficacy of sunitinib in patients with advanced HCC. In the study by Zhu et al (2007) patients with advanced HCC received sunitinib $37.5 \mathrm{mg}$ once daily on a standard 4-weekson/2-weeks-off regimen (6-week cycle). PFS was the primary endpoint. Median PFS in this trial was 4.1 months. A total of 26 patients were enrolled. Adverse events generally were manageable, and the most common adverse events included neutropenia, lymphopenia, thrombocytopenia, elevation of transaminases, fatigue, and skin rash. In the other phase 2 study, sunitinib was administered at a dose of $50 \mathrm{mg}$ daily in a 4-weeks-on/2-weeks-off regimen to patients with unresectable HCC (Faivre et al 2007). The primary endpoint of the study was the overall response rate according to RECIST criteria. Thirty-seven patients entered the trial, 1 patient had a confirmed PR, and 39\% of patients had SD. Grade 3 and 4 toxicities included thrombocytopenia (43\%), neutropenia (24\%), central nervous system symptoms (24\%), asthenia (22\%), and hemorrhage (14\%). Of interest, in a significant number of patients sunitinib led to tumor necrosis in both studies; however, whether tumor necrosis is indeed correlated with clinical efficacy remain to be defined in future studies.

\section{mTOR inhibition}

In vitro as well as preclinical in vivo data of HCC show that mTOR inhibition by rapamycin and its analogues (the cell cycle inhibitor-779 [CCI-779, temsirolimus], RAD001 [everolimus]) an orally bioavailable derivative of rapamycin, AP23573) significantly reduces HCC growth and improves survival primarily via antiangiogenic effects (Sahin et al 2004). A phase 2/3 trial evaluating everolimus for advanced $\mathrm{HCC}$ is currently starting to recruit patients (Rizell and Lindner 2005) (NIH, NCT00390195). In addition, the combination treatment of rapamycin and its analalogues with conventional cytostatic drugs (eg, doxorubicin, vinblastine) has been shown to enhance the antitumoral potency compared with monotherapy with either doxorubicin or vinblastine in patients with HCC (Ribatti et al 2007; Semela et al 2007; Sieghart et al 2007). Therefore, the in vitro and in vivo data from other tumor entities suggest that mTOR inhibitors, including the rapamycin analogues CCI-779, RAD001 and AP23573, are promising combination agents for future cancer therapy.

\section{Proteasome inhibition}

Bortezomib $\left(\right.$ Velcade $\left.^{\circledR}\right)$ is a proteasome inhibitor, which blocks multi-ubiquitinated protein degradation by reversibly and competitively inhibiting the active site threonine residue of the 26S proteasome (Mitsiades et al 2005). Antineoplastic activity of bortezomib has already beeen shown in several in vitro and in vivo studies (Brignole et al 2006; Baradari et al 2007; Hopfner et al 2008) and has been recently approved for the treatment of mantle cell lymphoma. A phase 1/2 trial of bortezomib in patients with advanced HCC showed disease stabilization in some patients with good tolerability. In this study the authors recommend evaluating combination treatment strategies using bortezomib together with HCC-relevant cytostatics such as doxorubicin (Hegewisch-Becker et al 2004).

\section{Conclusion}

Agents that specifically target key molecules in carcinogenesis have emerged over the last decade. Of major interest are the growth factors and their receptors as well as their signaling pathways. The molecular targeting of these factors has become a promising approach for the effective treatment of various cancers, including hepatocellular carcinoma. The recent in vitro and in vivo findings as well as the clinical trials clearly demonstrate that advanced HCC is no longer a tumor without specific medical treatment options. Sorafenib, a multi-kinase inhibitor, is the first medication that is now approved for the treatment of patients with advanced HCC. However, several other promising new drugs are currently under investigation. Most probably, the combination of agents with different molecular targets as the growth factor receptor inhibitors, proteasome inhibitors, or cytostatics will improve the clinical response in patients with advanced $\mathrm{HCC}$ with tolerable and manageable side effects. Furthermore, the use of targeted therapies such as sorafenib, that are already approved for therapy in advanced HCC, might also improve HCC recurrence in patients who undergo resection, local ablation and transarterial chemoembolization, which is currently being tested in several studies.

\section{Disclosures}

None of the authors has any conflicts of interest to disclose.

\section{References}

Abou-Alfa GK, Schwartz L, Ricci S, et al. 2006. Phase II study of sorafenib in patients with advanced hepatocellular carcinoma. J Clin Oncol, 24:4293-300.

Akuta N, Suzuki F, Kawamura Y, et al. 2007. Amino acid substitutions in the hepatitis $\mathrm{C}$ virus core region are the important predictor of hepatocarcinogenesis. Hepatology, 46:1357-64.

Alberts SR MB, Kim GP, et al. 2007. NCCTG phase II trial (N044J) of AZD2171 for patients with hepatocellular carcinoma (HCC)-interim review of toxicity [abstract]. Gastrointestinal Cancer Symposium, Orlando, Fl, Abstract 186. 
Arora A, Scholar EM. 2005. Role of tyrosine kinase inhibitors in cancer therapy. J Pharmacol Exp Ther, 315:971-9.

Balkwill F, Coussens LM. 2004. Cancer: an inflammatory link. Nature, 431:405-6.

Baradari V, Hopfner M, Huether A, et al. 2007. Histone deacetylase inhibitor MS-275 alone or combined with bortezomib or sorafenib exhibits strong antiproliferative action in human cholangiocarcinoma cells. World $J$ Gastroenterol, 13:4458-66.

Befeler AS, Di Bisceglie AM. 2002. Hepatocellular carcinoma: diagnosis and treatment. Gastroenterology, 122:1609-19.

Bourhis J, Rivera F, Mesia R, et al. 2006. Phase I/II study of cetuximab in combination with cisplatin or carboplatin and fluorouracil in patients with recurrent or metastatic squamous cell carcinoma of the head and neck. J Clin Oncol, 24:2866-72.

Bravi F, Bosetti C, Tavani A, et al. 2007. Coffee drinking and hepatocellular carcinoma risk: a meta-analysis. Hepatology, 46:430-5.

Brechot C. 2004. Pathogenesis of hepatitis B virus-related hepatocellular carcinoma: old and new paradigms. Gastroenterology, 127:S56-61.

Brignole C, Marimpietri D, Pastorino F, et al. 2006. Effect of bortezomib on human neuroblastoma cell growth, apoptosis, and angiogenesis. $J$ Natl Cancer Inst, 98:1142-57.

Bruix J, Llovet JM. 2002. Prognostic prediction and treatment strategy in hepatocellular carcinoma. Hepatology, 35:519-24.

Bruix J, Sala M, Llovet JM. 2004. Chemoembolization for hepatocellular carcinoma. Gastroenterology, 127:S179-88.

Bruix J, Sherman M. 2005. Management of hepatocellular carcinoma. Hepatology, 42:1208-36.

Bruix J, Sherman M, Llovet JM, et al. 2001. Clinical management of hepatocellular carcinoma. Conclusions of the Barcelona-2000 EASL conference. European Association for the Study of the Liver. J Hepatol, 35:421-30.

Bruno S, Crosignani A, Maisonneuve P, et al. 2007. Hepatitis C virus genotype $1 \mathrm{~b}$ as a major risk factor associated with hepatocellular carcinoma in patients with cirrhosis: a seventeen-year prospective cohort study. Hepatology. 46:1350-6.

Busby JE, Kim SJ, Yazici S, et al. 2006. Therapy of multidrug resistant human prostate tumors in the prostate of nude mice by simultaneous targeting of the epidermal growth factor receptor and vascular endothelial growth factor receptor on tumor-associated endothelial cells. Prostate, 66:1788-98.

Caldwell SH, Crespo DM, Kang HS, et al. 2004. Obesity and hepatocellular carcinoma. Gastroenterology, 127:S97-103.

Calle EE, Rodriguez C, Walker-Thurmond K, et al. 2003. Overweight, obesity, and mortality from cancer in a prospectively studied cohort of U.S. adults. $N$ Engl J Med, 348:1625-38.

Calvisi DF, Ladu S, Gorden A, et al. 2007. Mechanistic and prognostic significance of aberrant methylation in the molecular pathogenesis of human hepatocellular carcinoma. J Clin Invest, 117:2713-22.

Campbell JS, Hughes SD, Gilbertson DG, et al. 2005. Platelet-derived growth factor $\mathrm{C}$ induces liver fibrosis, steatosis, and hepatocellular carcinoma. Proc Natl Acad Sci U S A, 102:3389-94.

Capocaccia R, Sant M, Berrino F, et al. 2007. Hepatocellular carcinoma: trends of incidence and survival in Europe and the United States at the end of the 20th century. Am J Gastroenterol, 102:1661-1670. quiz 1660, 1671.

Chao Y, Li CP, Chau GY, et al. 2003. Prognostic significance of vascular endothelial growth factor, basic fibroblast growth factor, and angiogenin in patients with resectable hepatocellular carcinoma after surgery. Ann Surg Oncol, 10:355-62.

Chen CJ, Chen DS. 2002. Interaction of hepatitis B virus, chemical carcinogen, and genetic susceptibility: multistage hepatocarcinogenesis with multifactorial etiology. Hepatology, 36:1046-9.

Chen CJ, Yang HI, Su J, et al. 2006. Risk of hepatocellular carcinoma across a biological gradient of serum hepatitis B virus DNA level. JAMA, 295:65-73.

Chevret S, Trinchet JC, Mathieu D, et al. 1999. A new prognostic classification for predicting survival in patients with hepatocellular carcinoma. Groupe d'Etude et de Traitement du Carcinome Hepatocellulaire. J Hepatol, 31:133141.
Deuffic S, Poynard T, Buffat L, et al. 1998. Trends in primary liver cancer. Lancet, 351:214-15.

Di Bisceglie AM. 2004. Screening for hepatocellular carcinoma: being old is not all bad. Am J Gastroenterol, 99:1477-8.

Diehl AM. 2002. Liver regeneration. Front Biosci, 7:e301-14.

Dufour JF, Huber O, Kozma SC, et al. 2007. Tumour suppressors in liver carcinogenesis. J Hepatol, 47:860-7.

Duncan SA. 2003. Mechanisms controlling early development of the liver. Mech Dev, 120:19-33

El-Serag HB. 2004. Hepatocellular carcinoma: recent trends in the United States. Gastroenterology, 127:S27-34.

El-Serag HB, Davila JA, Petersen NJ, et al. 2003. The continuing increase in the incidence of hepatocellular carcinoma in the United States: an update. Ann Intern Med, 139:817-23.

El-Serag HB, Mason AC. 1999. Rising incidence of hepatocellular carcinoma in the United States. N Engl J Med, 340:745-50.

El-Serag HB, Rudolph KL. 2007. Hepatocellular carcinoma: epidemiology and molecular carcinogenesis. Gastroenterology, 132:2557-76.

El-Serag HB, Siegel AB, Davila JA, et al. 2006. Treatment and outcomes of treating of hepatocellular carcinoma among Medicare recipients in the United States: a population-based study. J Hepatol, 44:158-66.

El-Serag HB, Tran T, Everhart JE. 2004. Diabetes increases the risk of chronic liver disease and hepatocellular carcinoma. Gastroenterology, 126:460-8.

Faivre S, Ramiond E, Douillard J. 2007. Assessment of safety and druginduced tumor necrosis with sunitinib in patients with unresectable HCC. J Clin Oncol, 25:A4637.

Farinati F, Rinaldi M, Gianni S, et al. 2000. How should patients with hepatocellular carcinoma be staged? Validation of a new prognostic system. Cancer, 89:2266-73.

Fattovich G, Stroffolini T, Zagni I, et al. 2004. Hepatocellular carcinoma in cirrhosis: incidence and risk factors. Gastroenterology, 127:S35-50.

Gollob JA, Wilhelm S, Carter C, et al. 2006. Role of Raf kinase in cancer: therapeutic potential of targeting the Raf/MEK/ERK signal transduction pathway. Semin Oncol, 33:392-406.

Gruenwald V, Wilkens L, Gebel M. 2007. A phase II open-label study of cetuximab in unresectable hepatocellular carcinom: final results. J Clin Oncol, 25:A4594.

Hassan MM, Hwang LY, Hatten CJ, et al. 2002. Risk factors for hepatocellular carcinoma: synergism of alcohol with viral hepatitis and diabetes mellitus. Hepatology, 36:1206-13.

Hegewisch-Becker S, Sterneck M, Schubert U. 2004. Phase I/II trial of bortezomib in patients with unresectable hepatocellular carcinoma. $J$ Clin Oncol, 22:A4089.

Heymach JV. 2005. ZD6474 - clinical experience to date. Br J Cancer, 92(Suppl 1):S14-20.

Hopfner M, Schuppan D, Scherubl H. 2008. Growth factor receptors and related signalling pathways as targets for novel treatment strategies of hepatocellular cancer. World J Gastroenterol, 14:1-14.

Huether A, Hopfner M, Baradari V, et al. 2005a. EGFR blockade by cetuximab alone or as combination therapy for growth control of hepatocellular cancer. Biochem Pharmacol, 70:1568-1578.

Huether A, Hopfner M, Sutter AP, et al. 2005b. Erlotinib induces cell cycle arrest and apoptosis in hepatocellular cancer cells and enhances chemosensitivity towards cytostatics. J Hepatol, 43:661-9.

Jeng KS, Sheen IS, Wang YC, et al. 2004. Prognostic significance of preoperative circulating vascular endothelial growth factor messenger RNA expression in resectable hepatocellular carcinoma: a prospective study. World J Gastroenterol, 10:643-6.

Kiyosawa K, Umemura T, Ichijo T, et al. 2004. Hepatocellular carcinoma: recent trends in Japan. Gastroenterology, 127:S17-26.

Koch I, Baron A, Roberts S. 2005. Influence of hepatic dysfunction on safety, tolerability and pharmacocinetics of PTK787/ZK222584 in patients with unresectable HCC. J Clin Oncol, 23:A4134.

Kudo M, Chung H, Haji S, et al. 2004. Validation of a new prognostic staging system for hepatocellular carcinoma: the JIS score compared with the CLIP score. Hepatology, 40:1396-405. 
Kulik LM. 2007. Advancements in hepatocellular carcinoma. Curr Opin Gastroenterol, 23:268-74.

Leung TW, Tang AM, Zee B, et al. 2002. Construction of the Chinese University Prognostic Index for hepatocellular carcinoma and comparison with the TNM staging system, the Okuda staging system, and the Cancer of the Liver Italian Program staging system: a study based on 926 patients. Cancer, $94: 1760-9$.

Liu L, Cao Y, Chen C, et al. 2006. Sorafenib blocks the RAF/MEK/ERK pathway, inhibits tumor angiogenesis, and induces tumor cell apoptosis in hepatocellular carcinoma model PLC/PRF/5. Cancer Res, $66: 11851-8$

Llovet J, Rici S, Mazzaferro V, et al. 2007. Randomized phase III trial of sorafenib versus plazebo in patients with advanced hepatocellular carcinoma. J Clin Oncol, 25:LBA1.

Llovet JM, Bru C, Bruix J. 1999. Prognosis of hepatocellular carcinoma: the BCLC staging classification. Semin Liver Dis, 19:329-38.

Llovet JM, Burroughs A, Bruix J. 2003. Hepatocellular carcinoma. Lancet, 362:1907-17.

Llovet JM, Fuster J, Bruix J. 2004. The Barcelona approach: diagnosis, staging, and treatment of hepatocellular carcinoma. Liver Transpl, 10:S115-20.

Louafi S, Hebbar M, Rosmorduc O. 2007. Gemcitabine, oxalaplatin and cetuximab for treatment of hepatocellular carcinoma: results of phase II study ERGO. J Clin Oncol, : 18S.

Marrero JA. 2006. Hepatocellular carcinoma. Curr Opin Gastroenterol, 22:248-53.

Marrero JA, Fontana RJ, Barrat A, et al. 2005a. Prognosis of hepatocellular carcinoma: comparison of 7 staging systems in an American cohort Hepatology, 41:707-16.

Marrero JA, Fontana RJ, Fu S, et al. 2005b. Alcohol, tobacco and obesity are synergistic risk factors for hepatocellular carcinoma. $J$ Hepatol, 42:218-24.

Mendel DB, Laird AD, Xin X, et al. 2003. In vivo antitumor activity of SU11248, a novel tyrosine kinase inhibitor targeting vascular endothelial growth factor and platelet-derived growth factor receptors: determination of a pharmacokinetic/pharmacodynamic relationship. Clin Cancer Res, 9:327-37.

Ming L, Thorgeirsson SS, Gail MH, et al. 2002. Dominant role of hepatitis B virus and cofactor role of aflatoxin in hepatocarcinogenesis in Qidong, China. Hepatology, 36:1214-20.

Mitsiades CS, Mitsiades N, Hideshima T, et al. 2005. Proteasome inhibitors as therapeutics. Essays Biochem, 41:205-18.

Moore M, Goldstein D, Hamm J. 2005. Erlotinib improves survival when added to gemcitabine in patients with advanced pancreatic cancer. A phase III trial of the National Cancer Institute of Canada Clinical Trials. J Clin Oncol, 23:LBA1.

Morgan TR, Mandayam S, Jamal MM. 2004. Alcohol and hepatocellular carcinoma. Gastroenterology, 127:S87-96.

Nakamoto Y, Guidotti L, Kuhlen C, et al. 1998. Immune pathogenesis of hepatocellular carcinoma. $J$ Exp Med, 188:341-50.

Nakamoto Y, Kaneko S, Fan H, et al. 2002. Prevention of hepatocellular carcinoma development associated with chronic hepatitis by anti-fas ligand antibody therapy. J Exp Med, 196:1105-11.

Naugler WE, Sakurai T, Kim S, et al. 2007. Gender disparity in liver cancer due to sex differences in MyD88-dependent IL-6 production. Science, 317:121-4.

O'Dwyer PJ, Giantonio BJ, Levy DE, et al. 2006. Gefitinib in advanced unresectable hepatocellular carcinoma: results from the Eastern Cooperative Oncology Groups's study E1203. J Clin Oncol, 24:A4143.

Ogasawara S, Yano H, Iemura A, et al. 1996. Expressions of basic fibroblast growth factor and its receptors and their relationship to proliferation of human hepatocellular carcinoma cell lines. Hepatology, 24:198-205.

Ohata K, Hamasaki K, Toriyama K, et al. 2003. Hepatic steatosis is a risk factor for hepatocellular carcinoma in patients with chronic hepatitis C virus infection. Cancer, 97:3036-43

Okuda K. 2000.Hepatocellular carcinoma. J Hepatol, 32:225-37.
Okuda K, Ohtsuki T, Obata H, et al. 1985. Natural history of hepatocellular carcinoma and prognosis in relation to treatment. Study of 850 patients. Cancer, 56:918-28.

Ozturk M. 1999. Genetic aspects of hepatocellular carcinogenesis. Semin Liver Dis, 19:235-42,

Pawson T. 2002. Regulation and targets of receptor tyrosine kinases. Eur $J$ Cancer, 38(Suppl 5):S3-10

Philip PA, Mahoney MR, Allmer C, et al. 2005. Phase II study of Erlotinib (OSI-774) in patients with advanced hepatocellular cancer. J Clin Oncol, 23:6657-63.

Pikarsky E, Porat RM, Stein I, et al. 2004. NF-kappaB functions as a tumour promoter in inflammation-associated cancer. Nature, 431:461-6.

Pollicino T, Squadrito G, Cerenzia G, et al. 2004. Hepatitis B virus maintains its pro-oncogenic properties in the case of occult HBV infection. Gastroenterology, 126:102-10.

Poon RT, Ho JW, Tong CS, et al. 2004. Prognostic significance of serum vascular endothelial growth factor and endostatin in patients with hepatocellular carcinoma. Br J Surg, 91:1354-60.

Ramanathan RK, Belani CP, Singh DA. 2006. Phase II study of lapatinib, a dual inhibitor of epidermal growth factor receptor tyrosine kinase 1 and $2(\mathrm{Her} 2 / \mathrm{Neu})$ in patients with advanced biliary tree cancer or hepatocellular carcinoma. J Clin Oncol, 24:A4010.

Ribatti D, Nico B, Mangieri D, et al. 2007. In vivo inhibition of human hepatocellular carcinoma related angiogenesis by vinblastine and rapamycin. Histol Histopathol, 22:285-9.

Rizell M, Lindner P. 2005. Inhibition of mTOR suppresses experimental liver tumours. Anticancer Res, 25:789-93.

Sahin F, Kannangai R, Adegbola O, et al. 2004. mTOR and P70 S6 kinase expression in primary liver neoplasms. Clin Cancer Res, 10:8421-5.

Sangiovanni A, Del Ninno E, Fasani P, et al. 2004. Increased survival of cirrhotic patients with a hepatocellular carcinoma detected during surveillance. Gastroenterology, 126:1005-14.

Satyanarayana A, Manns MP, Rudolph KL. 2004. Telomeres and telomerase: a dual role in hepatocarcinogenesis. Hepatology, 40:276-83.

Schwartz J, Schwartz M, Lehrer D. 2006. Bevacizumab in unresectable hepatocellular carcinoma for patients without metastasis and without invasion of the portal vein. J Clin Oncol, 24:A4144.

Semela D, Piguet AC, Kolev M, et al. 2007. Vascular remodeling and antitumoral effects of mTOR inhibition in a rat model of hepatocellular carcinoma. $J$ Hepatol, 46:840-8.

Sherman M, Klein A. 2004. AASLD single-topic research conference on hepatocellular carcinoma: Conference proceedings. Hepatology, 40:1465-73.

Sieghart W, Fuereder T, Schmid K, et al. 2007. Mammalian target of rapamycin pathway activity in hepatocellular carcinomas of patients undergoing liver transplantation. Transplantation, 83:425-32.

Sun HC, Zhang W, Qin LX, et al. 2007. Positive serum hepatitis B e antigen is associated with higher risk of early recurrence and poorer survival in patients after curative resection of hepatitis B-related hepatocellular carcinoma. J Hepatol, 47:684-90.

Suriawinata A, Xu R. 2004. An update on the molecular genetics of hepatocellular carcinoma. Semin Liver Dis, 24:77-88.

Talwalkar JA, Gores GJ. 2004. Diagnosis and staging of hepatocellular carcinoma. Gastroenterology, 127:S126-32.

Taylor-Robinson SD, Foster GR, Arora S, et al. 1997. Increase in primary liver cancer in the UK, 1979-1994. Lancet, 350:1142-3.

Thomas M, Chadha R, Iwasak M. 2007. The combination of bevacizumab and erlotinib shows significant biological activity in patients with advanced HCC. J Clin Oncol, 25:A15190.

Thomas MB, Chadha R, Glover K, et al. 2007. Phase 2 study of erlotinib in patients with unresectable hepatocellular carcinoma. Cancer, 110:1059-67.

Velazquez RF, Rodriguez M, Navascues CA, et al. 2003. Prospective analysis of risk factors for hepatocellular carcinoma in patients with liver cirrhosis. Hepatology, 37:520-7. 
Wiedmann M, Feisthammel J, Bluthner T, et al. 2006. Novel targeted approaches to treating biliary tract cancer: the dual epidermal growth factor receptor and ErbB-2 tyrosine kinase inhibitor NVP-AEE788 is more efficient than the epidermal growth factor receptor inhibitors gefitinib and erlotinib. Anticancer Drugs, 17:783-95.

Wilhelm SM, Carter C, Tang L, et al. 2004. BAY 43-9006 exhibits broad spectrum oral antitumor activity and targets the RAF/MEK/ERK pathway and receptor tyrosine kinases involved in tumor progression and angiogenesis. Cancer Res, 64:7099-109.

Yang HI, Lu SN, Liaw YF, et al. 2002. Hepatitis B e antigen and the risk of hepatocellular carcinoma. $N$ Engl J Med, 347:168-74.

Yano Y, Yamashita F, Sumie S, et al. 2002. Clinical features of hepatocellular carcinoma seronegative for both HBsAg and anti-HCV antibody but positive for anti-HBc antibody in Japan. Am J Gastroenterol, 97:156-61.
Younes MN, Park YW, Yazici YD, et al. 2006. Concomitant inhibition of epidermal growth factor and vascular endothelial growth factor receptor tyrosine kinases reduces growth and metastasis of human salivary adenoid cystic carcinoma in an orthotopic nude mouse model. Mol Cancer Ther, 5:2696-705.

Yu MC, Yuan JM. 2004. Environmental factors and risk for hepatocellular carcinoma. Gastroenterology, 127:S72-8.

Zhu AX. 2008. Development of sorafenib and other molecularly targeted agents in hepatocellular carcinoma. Cancer, 112:250-9.

Zhu AX, Sahani D, di Tomasi E. 2007. A phase II study of sutininib in patients with advanced HCC. J Clin Oncol, 25:A4637.

Zhu AX, Stuart K, Blaszkowsky LS, et al. 2007. Phase 2 study of cetuximab in patients with advanced hepatocellular carcinoma. Cancer, 110:581-9. 